\title{
Bounds on partial ovoids and spreads in classical generalized quadrangles
}

\author{
Stefaan De Winter* $\quad$ Koen Thas ${ }^{\dagger}$
}

\begin{abstract}
We present an improvement on a recent bound for small maximal partial ovoids of $\mathbf{W}\left(q^{3}\right)$. We also classify maximal partial ovoids of size $\left(q^{2}-1\right)$ of $\mathbf{Q}(4, q)$ which allow a certain large automorphism group, and discuss examples for small $q$.
\end{abstract}

Keywords : partial ovoid, partial spread, generalized quadrangle

MSC 2000: 51E23, 51E12

\section{Introduction}

Generalized polygons were introduced in 1959 by Tits in the appendix of his celebrated paper on triality [23]. Ever since they have played a key role in algebraic and combinatorial geometry. Among the generalized polygons, the finite generalized quadrangles (GQs) occupy a special place, in particular because of the existence of several classical and non-classical examples which can be studied in a geometric, algebraic as well as combinatorial way; they are connected with a broad collection of other interesting objects from Galois geometry and algebra, such as polarities, (pseudo-)ovals, (pseudo-)ovoids, $q$-clans, flocks, fans, fibrations, herds, 4-gonal families, BN-pairs, ... Since their introduction several authors have been interested in the study of substructures of finite GQs, in particular in the existence of spreads and ovoids (see further for a formal introduction to these objects). As there already is an extensive literature on this subject containing some good surveys, we will only tersely overview some of the most

\footnotetext{
${ }^{*}$ The first author is a Postdoctoral Fellow of the Research Foundation - Flanders (FWO).

${ }^{\dagger}$ The second author is partially supported by the Research Foundation - Flanders (FWO).
} 




\section{Small maximal partial spreads and ovoids}

Theorem 4.1 ([4]). (i) The second smallest maximal partial ovoids $\mathcal{K}$ of $\mathbf{W}\left(q^{2}\right)$, $q=p^{h}, p>3$ prime, $h \geq 1$, contain at least $s\left(q^{2}\right)+1$ points, where $s\left(q^{2}\right)$ denotes the cardinality of the second smallest non-trivial minimal blocking sets in $\mathrm{PG}\left(2, q^{2}\right)$. If $q=p>2$, then $\mathcal{K}$ contains at least $3\left(p^{2}+1\right) / 2+1$ points.

(ii) The second smallest maximal partial ovoids $\mathcal{K}$ of $\mathbf{W}\left(q^{3}\right), q=p^{h}, p \geq 7$ prime, $h \geq 1$, contain at least $q^{3}+q^{2}+q+1$ points. If $|\mathcal{K}|=q^{3}+q^{2}+q+1$, then $\mathcal{K}$ consists of the point set of a subgeometry $\mathrm{PG}(3, q)$ of $\operatorname{PG}\left(3, q^{3}\right)$.

In [4] the existence of maximal partial ovoids of $\mathbf{W}\left(q^{3}\right)$ of size $q^{3}+q^{2}+q+1$ was posed as an open problem. It was J. A. Thas who suggested to the authors to try to use the Klein correspondence in order to prove the (non-)existence of such a partial ovoid. We will now use this correspondence to prove its nonexistence. We mention the following lemma which will be used without further notice.

Lemma 4.2. Consider a hyperplane $\pi$ of $\mathrm{PG}\left(5, q^{3}\right)$ and a subspace $\Omega:=\mathrm{PG}(5, q)$ of $\mathrm{PG}\left(5, q^{3}\right)$. Then $\pi$ intersects $\Omega$ in at least a plane $\mathrm{PG}(2, q)$.

Theorem 4.3. The GQ $\mathbf{W}\left(q^{3}\right), q=p^{h}, p \geq 7$, does not admit a maximal partial ovoid of size $q^{3}+q^{2}+q+1$.

Proof. Assume that $\mathbf{W}\left(q^{3}\right)$ admits a maximal partial ovoid $\mathcal{K}$ of size $q^{3}+q^{2}+$ $q+1$. We consider $\mathbf{W}\left(q^{3}\right)$ in its natural representation in $\operatorname{PG}\left(3, q^{3}\right)$. Then $\mathcal{K}$ corresponds to some $\mathrm{PG}(3, q)$-subgeometry of $\mathrm{PG}\left(3, q^{3}\right)$. Using the Klein correspondence the lines of $\mathrm{PG}\left(3, q^{3}\right)$ are mapped onto the points of $\mathbf{Q}^{+}\left(5, q^{3}\right)$, the lines of $\mathrm{PG}(3, q)$ onto the points of some $\mathbf{Q}^{+}(5, q) \subset \mathbf{Q}^{+}\left(5, q^{3}\right)$ and the lines of $\mathbf{W}\left(q^{3}\right)$ onto the points of some $\mathbf{Q}\left(4, q^{3}\right) \subset \mathbf{Q}^{+}\left(5, q^{3}\right)$. It is easily seen, since $\mathcal{K}$ is a partial ovoid, that $\mathbf{Q}\left(4, q^{3}\right) \cap \mathbf{Q}^{+}(5, q)=\emptyset$ (as point sets). However, the $\mathrm{PG}\left(4, q^{3}\right)$ determined by $\mathbf{Q}\left(4, q^{3}\right)$ and the $\mathrm{PG}(5, q)$ determined by $\mathbf{Q}^{+}(5, q)$ must intersect in at least a plane $\mathrm{PG}(2, q)$. As in $\mathrm{PG}(5, q)$ every plane has nonempty intersection with $\mathbf{Q}^{+}(5, q), \mathcal{K}$ cannot be a partial ovoid.

Concerning small maximal partial ovoids of $\mathbf{W}(q)$, it is interesting to note that computer searches [4], exhaustive for $q \in\{2,3,4,5\}$ and heuristic for $q \in$ $\{7,8,9,11,13,16,17,19,23,25,27\}$, suggest that the second smallest maximal partial ovoids of $\mathbf{W}(q)$ will probably have size $2 q+1$. A maximal partial ovoid of this size can easily be constructed by taking all points except one point $r$ on a hyperbolic line $H$ in $\mathbf{W}(q)$, together with one arbitrary point (not collinear with one of the remaining points of $H$ ) from each of the $q+1$ lines of $\mathbf{W}(q)$ 

We now turn to small maximal partial spreads. Since $\mathbf{H}\left(3, q^{2}\right)$ is the only subGQ of order $\left(q^{2}, q\right)$ of $\mathbf{H}\left(4, q^{2}\right)$ and $\mathbf{H}\left(3, q^{2}\right)$ does not admit a spread, it follows that a smallest maximal partial spread of $\mathbf{H}\left(4, q^{2}\right)$ contains at least $q^{3}+2$ lines. (Note that if the size would have been $q^{3}+1$, the points on the lines of the partial spread would form the point set of a subGQ of order $\left(q^{2}, q\right)$.) However using an analogous technique as was used in [2] to show the non-existence of a maximal partial spread of size $q^{2}+2$ of $\mathbf{Q}(5, q)$, we can easily exclude the existence of such a maximal partial spread.

full screen

close

Theorem 4.7. The GQ $\mathbf{H}\left(4, q^{2}\right)$ does not admit a maximal partial spread of size $q^{3}+2$.

Proof. Suppose that $\mathcal{M}$ is a maximal partial spread of size $q^{3}+2$, and let $X$ be the set of points of $\mathbf{H}\left(4, q^{2}\right)$ not covered by $\mathcal{M}$. Then $|X|=q^{7}-q^{3}-q^{2}-$ 1. Further, through every point of $X$ there is a unique line intersecting $\overline{\mathcal{M}}$ in exactly 2 points (while all other lines through such a point intersect $\widetilde{\mathcal{M}}$ in 1 point). Consequently the number of lines intersecting $\widetilde{\mathcal{M}}$ in 2 points equals $|X| /\left(q^{2}-1\right)$. This quantity can never be an integer, a contradiction.

The best known result is the following.

Theorem 4.8 ([12]). A maximal partial spread of the $G Q \mathbf{H}\left(4, q^{2}\right)$ has more than $q^{3}+q \sqrt{q}-q / 2-3 / 8 \sqrt{q}+7 / 8$ lines.

\section{Partial ovoids of $\mathrm{Q}(4, q)$ of size $q^{2}-1$ for $q \in\{2,3,5,7,11\}$, and beyond}

Partial ovoids of $\mathbf{Q}(4, q)$ of size $q^{2}-1$ are only known for $q \in\{2,3,5,7,11\}$. For $q=2$ there is, up to isomorphism, a unique 3 -arc of $\mathbf{Q}(4,2)$, see, e.g., [21]. For $q=3$ an 8-arc can easily be constructed as follows. Consider any elliptic quadric $\mathbf{Q}^{-}(3,3) \subset \mathbf{Q}(4,3)$ and any point $p \notin \mathbf{Q}^{-}(3,3)$. Then $p$ is collinear with exactly 4 points of $\mathbf{Q}^{-}(3,3)$, and these points determine a conic $C$. The set $C^{\perp}$ contains two points (among which $p$ ). It is easily seen that $\left(\mathbf{Q}^{-}(3,3) \backslash C\right) \cup C^{\perp}$ is a complete 8-arc of $\mathbf{Q}(4,3)$. It has longtime been thought that these were the only values of $q$ for which $\left(q^{2}-1\right)$-arcs of $\mathbf{Q}(4, q)$ existed. However in 2003 Penttila disproved this by showing with the computer the existence of $\left(q^{2}-1\right)$ arcs of $\mathbf{Q}(4, q)$ for $q \in\{5,7,11\}$ [14]. In [4] Cimrakova and Fack confirmed these results, also with the use of the computer. Their searches were heuristic and so by no means exclude the existence of $\left(q^{2}-1\right)$-arcs of $\mathbf{Q}(4, q)$ for other values of odd $q$. It is important to note that no computer free constructions are known of $\left(q^{2}-1\right)$-arcs for $q \in\{7,11\}$. It is however possible to describe 

The previous theorem implies that in order to construct a 24 -arc in $\mathbf{Q}(4,5)$ it is sufficient to find 4 doubly subtended conics in some $\mathbf{Q}(3,5) \subset \mathbf{Q}(4,5)$ which intersect two by two in exactly 2 points. In order to do so consider $\mathbf{Q}(4,5)$ in $\mathrm{PG}(4,5)$ determined by $X_{0}^{2}+X_{1} X_{2}+X_{3} X_{4}=0$ and the $\mathbf{Q}(3,5) \subset \mathbf{Q}(4,5)$ in the hyperplane $X_{0}=0$. Then the conics in the hyperplane $X_{0}=0$ with equations

$$
\begin{array}{cc}
C_{1}:\left\{\begin{array}{c}
X_{1}=X_{2} \\
X_{1}^{2}+X_{3} X_{4}=0
\end{array}\right. & C_{2}:\left\{\begin{array}{c}
X_{1}=-X_{2} \\
-X_{1}^{2}+X_{3} X_{4}=0
\end{array}\right. \\
C_{3}:\left\{\begin{array}{c}
X_{3}=X_{4} \\
X_{3}^{2}+X_{1} X_{2}=0
\end{array}\right. & C_{4}:\left\{\begin{array}{c}
X_{3}=-X_{4} \\
-X_{3}^{2}+X_{1} X_{2}=0
\end{array}\right.
\end{array}
$$

are quickly seen to be doubly subtended and satisfy the desired property (i.e. $\left|C_{i} \cap C_{j}\right|=2$ if $i \neq j$ ). Consequently we have constructed a 24 -arc in $\mathbf{Q}(4,5)$.

One might wonder whether it is possible to generalize this construction for other values of $q$, and at least in theory this seems to be possible for $q \in\{7,11\}$. Suppose that for $q=7$ we find a set of 12 doubly subtended conics $C_{1}, \ldots, C_{12}$ in $\mathbf{Q}(3,7) \subset \mathbf{Q}(4,7)$ such that $\left|C_{i} \cap C_{j}\right| \in\{0,2\}, C_{1}, \ldots, C_{12}$ cover exactly 32 points of $\mathbf{Q}(3,7)$ and such that each of these 32 points is contained in exactly 3 conics $C_{i}$. Then a construction analogous to the one for $q=5$ would yield a 48-arc of $\mathbf{Q}(4,7)$. However it is not clear whether such a set of conics exists. Finally, also for $q=11$ one could obtain a generalization (60 conics covering completely $\mathbf{Q}(3,11) \subset \mathbf{Q}(4,11))$, but here as well the existence of such a set is not known. It may be interesting to study the link between such sets of conics and (partial) flocks of $\mathbf{Q}(3, q)$ (cf. [19]).

Tim Penttila has noted to us in a private communication [14] that the examples $\mathcal{K}$ of complete $\left(q^{2}-1\right)$-arcs of $\mathbf{Q}(4, q)$ which were constructed by him for $q=5,7,11$ all satisfy the following property:

$$
\left(q^{2}-1\right)^{2} \text { divides the size of } \operatorname{Aut}(\mathbf{Q}(4, q))_{\mathcal{K}} \text {. }
$$

We end our paper by showing that, conversely, if such an arc satisfies (*), we necessarily have $q \in\{5,7,11\}$. We do not consider the case $q=3$, as then all maximal 8-arcs are known.

Recall Dickson's classification of the subgroups of $\operatorname{PSL}(2, q)$, with $q=p^{h}$, $p$ a prime (see [10, Hauptsatz 8.27, p. 213]); we list the possible subgroups $H \leq \operatorname{PSL}(2, q)$, as follows:

(i) $H$ is an elementary abelian $p$-group;

(ii) $H$ is a cyclic group of order $k$, where $k$ divides $(q \pm 1) / r$, where $r=$ $\operatorname{gcd}(q-1,2)$; 





[13] S. E. Payne and J. A. Thas, Finite Generalized Quadrangles, Res. Notes Math. 110, Pitman Advanced Publishing Program, Boston/London/Melbourne, 1984.

[14] T. Penttila, Complete $\left(q^{2}-1\right)$-arcs in $\mathbf{Q}(4, q), q=5,7,11$, Private communication, September 2003.

[15] G. Tallini, Blocking sets with respect to planes of $\mathrm{PG}(3, q)$ and maximal spreads of a non-singular quadric of $\mathbf{Q}(4, q)$, Mitt. Math. Sem. Giessen 201 (1991), 141-147.

[16] J. A. Thas, Ovoidal translation planes, Arch. Math. 23 (1972), 110-112.

[17] Semipartial geometries and spreads of classical polar spaces, $J$. Combin. Theory Ser. A 35 (1983), 58-66.

[18] 10 (1984), 135-144.

[19] _ Flocks, maximal exterior sets, and inversive planes, in Finite Geometries and Combinatorial Designs, Contemp. Math. 111 (1990), pp. 187-218.

[20] J. A. Thas and S. E. Payne, Spreads and ovoids of finite generalized quadrangles, Geom. Dedicata 52 (1994), 227-253.

[21] K. Thas, Nonexistence of complete $(s t-t / s)$-arcs in generalized quadrangles of order $(s, t)$, I, J. Combin. Theory Ser. A 97 (2002), 394-402.

[22] _ Symmetry in Finite Generalized Quadrangles, Front. Math. 1, Birkhäuser-Verlag, Basel-Boston-Berlin, 2004.

[23] J. Tits, Sur la trialité et certains groupes qui s'en déduisent, Ins. Hautes Etudes Sci. Publ. Math. 2 (1959), 13-60.

\section{Stefaan De Winter}

Department of Mathematics, Ghent University KrijgslaAn 281, S25, B-9000 Ghent, BelGIUM

e-mail: sgdwinte@me.com

Koen Thas

Department of Mathematics, Ghent University KrijgslaAn 281, S25, B-9000 Ghent, BeLGIUM

e-mail: kthas@cage.UGent.be 